\title{
INTERPOLATION FAILS FOR THE SOUSLIN-KLEENE CLOSURE OF THE OPEN SET QUANTIFIER LOGIC
}

\author{
J. A. SGRO ${ }^{1}$
}

\begin{abstract}
In this paper we show that the Souslin-Kleene closure of the open set quantifier logic fails to have interpolation. We also show that the notion of a $T_{0}$-topological space is not definable in this logic. This gives a more natural proof that it is strictly weaker than the interior operator logic.
\end{abstract}

The questions of whether the Souslin-Kleene closure of the open set quantifier logic is the interior operator logic or even has interpolation come naturally from the work of the author in [3], [4], and [5]. We begin by giving the formal definitions and then the three results which settle these questions.

Definition. The Souslin-Kleene closure, $\Delta\left(\mathcal{L}^{*}\right)$, of a logic $\mathcal{L}^{*}$ is the logic formed by adding the complementary pseudo-elementary classes to the elementary classes.

That is, $\Omega$ and $\Omega^{c}$ are $\operatorname{PC}_{\mathcal{L}^{*}}(L)$-classes if and only if they are $\operatorname{EC}_{\Delta\left(\mathcal{E}^{*}\right)}(L)$-classes. See [1] for further background.

Definition. Take a structure $\mathfrak{A}$ and $q \subseteq \mathscr{P}(A)$ and form $(\mathfrak{A}, q)$. If $q$ is a topology on $A$ then $(\mathfrak{A}, q)$ is called topological.

Definition. The open set quantifier logics $\mathcal{L}(Q)$ and $\mathcal{L}\left(Q^{n}\right)_{n \in \omega}$ are formed by adding quantifiers $Q x$ and $Q \vec{x}, n \in \omega$, to first order logic where the interpretations of $Q x \varphi(x)$ and $Q \vec{x} \varphi(\vec{x})$, respectively, are that the sets defined by $\varphi(x)$ and $\varphi(\vec{x})$ are open in the topology and the $n$th product topology. For further background see [3] and [4].

We are now ready to state and prove the main theorems of this paper.

THeOREM 1. For each $(\mathfrak{A}, q)$ where $\mathfrak{A}$ is an $L$-structure there is an $L^{\#} \supseteq L$ and an extension $\mathfrak{A}^{\#}$ of $\mathfrak{A}$ to $L^{\#}$ such that if $\left.(\mathfrak{B}, r) \equiv_{\mathfrak{C} Q}\right)\left(\mathfrak{U}^{\#}, q\right)$ then

$$
(\mathfrak{B} \uparrow L, r) \equiv_{\Delta(\mathfrak{L}(Q))}(\mathfrak{A}, q) \text {. }
$$

Proof. This result is a straightforward application of the definition of $\Delta(\mathcal{L}(Q))$.

CountereXAMPLE 2 . The counterexample to interpolation for $\Delta\left(\mathcal{L}\left(Q_{n \in \omega}^{n}\right)\right)$ is the same as the one for $\mathcal{L}(Q)$ as presented in [3] and [4].

We will assume that interpolation holds and derive a contradiction. Let $L_{1}=$ $\{B(x), C(x), R(x)\}$ and $L_{2}=\{B(x), C(x), P(x)\}$. We define $\varphi(R)$ to be

$$
\neg Q x B(x) \wedge \forall y(B(y) \leftrightarrow C(y) \vee R(y)) \wedge Q x R(x)
$$

Presented to the Society, February 26, 1979; received by the editors May 17, 1979.

AMS (MOS) subject classifications (1970). Primary 02H99; Secondary 02G99.

${ }^{1}$ This research was partially supported by NSF grant \# MCS-77-04131. 
and $\psi(P)$ to be

$$
\forall x(C(x) \rightarrow P(x)) \rightarrow \neg Q x(P(x) \wedge B(x)) .
$$

One easily sees that $\vDash \varphi(R) \rightarrow \psi(P)$. Take $A=\mathbf{N}$, i.e. the set of natural numbers

$$
B^{\mathfrak{A}}=\{2 n \mid n \in \mathrm{N}\}, \quad C^{\mathfrak{A}}=\{4 n \mid n \in \mathrm{N}\} .
$$

Define $(\mathfrak{A}, q)$ to be $\left\langle A, B^{\mathscr{A}}, C^{\mathscr{A}},\{\varnothing, \mathbf{N}\}\right\rangle$.

Now since we have assumed that $\Delta(\mathcal{L}(Q))$ has interpolation there is a $\theta \in$ $\Delta(\mathscr{L}(Q))$ such that $\vDash \varphi(R) \rightarrow \theta$ and $\vDash \neg \psi(P) \rightarrow \neg \theta$.

Without loss of generality we assume that $(\mathfrak{A}, q)$ models $\theta$ since the argument in the alternate case is entirely analogous.

Now expand $\mathfrak{A}$ to an $L^{\#}$-structure $\mathfrak{A}^{\#}$ as in Theorem 1 . We then will expand $q$ to a $q^{\#}$ and define a $P^{\mathfrak{X}^{*}}$ such that $\left(\mathfrak{H}^{\#}, q\right) \prec_{\mathfrak{L}(Q)}\left(\mathfrak{U}^{\#}, q^{\#}\right)$ and $\left(\mathfrak{A}, P^{\mathfrak{X}^{*}}, q^{\#}\right)$ F $\neg \psi(p)$. This implies that $\left(\mathfrak{A}, q^{\#}\right) \equiv_{\Delta(\mathscr{L}(Q))}(\mathfrak{A}, q)$ and $\left(\mathfrak{A}, q^{\#}\right) \vDash \neg \theta$ which is a contradiction.

Let $\psi_{i}(x), i \in \omega$, enumerate the $L_{A}^{\#}(Q)$ definable nonopen sets of $\left(\mathfrak{U}^{\#}, q\right)$. We proceed by induction. For 0 we pick an $x$ and $y$ such that

$$
y \in\left[\psi_{0}(x)\right]^{\left(\Re^{*}, q\right)}
$$

and

$$
x \in B^{\mathfrak{A} \#}-\left[\psi_{0}(x)\right]^{\left(\mathfrak{A}^{*}, q\right)}
$$

if possible, otherwise

$$
x \in A-\left[\psi_{0}(x)\right]^{\left(\mathscr{A}^{*}, q\right)} .
$$

Assume we have picked the sequences $y_{0}, \ldots, y_{n}$ and $x_{0}, \ldots, x_{n}$. We will now choose $x_{n+1}$ and $y_{n+1}$ as follows. Choose, if possible, $x \in B^{\mathfrak{x}^{*}}-\left[\psi_{n+1}(x)\right]^{\left(\mathfrak{x}^{*}, q\right)}$ such that $x \neq y_{i}$ for $0 \leqslant i \leqslant n$. Otherwise pick $y$ such that

$$
y \in\left[\psi_{n+1}(x)\right]^{\left(\Re^{*}, q\right)} \cap B^{\mathfrak{A}^{*}}
$$

and

$$
y \neq x_{i} \text { for } 0 \leqslant i \leqslant n .
$$

This is possible since otherwise $\left[\psi_{n+1}(x)\right]^{\left(\mathfrak{\Re}^{*}, q\right)} \cap B^{\mathfrak{x}^{*}}$ and $B^{\mathfrak{x}^{*}}-\left[\psi_{n+1}(x)\right]^{\left(\mathfrak{A}^{*}, q\right)}$ would be finite which would imply that $B^{\mathfrak{2}^{*}}$ is finite which is false.

Let $P^{\mathfrak{A}^{*}}$ be $\left(C^{\mathfrak{A}} \cup\left\{x_{i}\right\}_{i \in \omega}\right) \cap B^{\mathfrak{A} *}$ and let $q^{\#}$ be the topology generated by $q \cup\left\{P^{\mathfrak{X}^{*}}\right\}$. We claim that $\left(\mathfrak{U}^{\#}, q\right) \prec_{\mathfrak{Q}(Q)}\left(\mathfrak{U}^{\#}, q^{\#}\right)$ and that $\left(\mathfrak{U}^{\#}, P^{\mathfrak{X}^{*}}, q^{\#}\right)$ k $\neg \psi(P)$. The second clause is straightforward. We prove the first by induction on the number of occurrences of $Q x$.

If $\left(\mathfrak{U}^{\#}, q\right) \vDash Q x \varphi(x)$ then $\left(\mathfrak{A}^{\#}, q^{\#}\right) \vDash Q x \varphi(x)$ since $q \subseteq q^{\#}$, thus assume $\left(\mathfrak{U}^{\#}, q^{\#}\right)$ $\vDash Q x \varphi(x)$ and $\left(\mathfrak{I}^{\#}, q\right) \vDash \neg Q x \varphi(x)$ and derive a contradiction. Thus $[\varphi(x)]^{\left(\mathscr{A}^{*}, q\right)}=$ $P^{\mathfrak{A}^{*}}$. But there is a $k$ such that $[\varphi(x)]^{\left(\mathfrak{A}^{*}, q\right)}=\left[\psi_{k}(x)\right]^{\left(\mathfrak{A}^{*}, q\right)}$ so by the definition of $P^{\mathfrak{X}^{*}}$ either

$$
x_{k} \in P^{\mathfrak{A}^{*}}-\left[\psi_{k}(x)\right]^{\left(\mathfrak{A}^{*}, q\right)}
$$


or

$$
y_{k} \in\left[\psi_{k}(x)\right]^{\left(\mathscr{X}^{*}, q\right)}-P^{\mathscr{x}^{*}}
$$

Hence a contradiction.

REMARK. The analogous result for $\Delta\left(\mathcal{L}\left(Q^{n}\right)_{n \in \omega}\right)$ can be proved by the same method. Also this result shows that the interior operator logics $\mathcal{L}(I)$ and $\mathcal{L}\left(I^{n}\right)_{n \in \omega}$ as defined in [3] and [5] strictly contain $\Delta(\mathcal{L}(Q))$ and $\Delta\left(\mathcal{L}\left(Q^{n}\right)_{n \in \omega}\right)$, respectively, since they both have interpolation by [5].

By [2] we know that because $\Delta(\mathcal{L}(Q))$ and $\Delta\left(\mathcal{L}\left(Q^{n}\right)_{n \in \omega}\right)$ do not have interpolation they do not have a Beth definability theorem.

However this result of strict containment can be improved by giving a more natural counterexample in the topological sense.

Definition. A topological space is called $T_{0}$ (Minkowski), if and only if for each $x \neq y$ there is an open set containing one but not the other.

We can equivalently define a $T_{0}$-space as a space where unequal points have unequal closures. See [6].

The class of $T_{0}$-spaces is the class of models of the $\mathcal{L}(I)$ sentence

$$
\forall x \forall y(x \neq y \rightarrow(I y(y \neq x) \vee I x(x \neq y))) \text {. }
$$

However we will now prove that the class of $T_{0}$ models is not a basic elementary class of $\Delta(\mathfrak{L}(Q))$.

Take $\mathfrak{A}$ to be ${ }^{2} \mathbf{N}=\{f \mid f:\{0,1\} \rightarrow \mathbf{N}\}$ and $L=\varnothing$.

Define a pseudometric by $d(x, y)=|x(0)-y(0)|$. Then the topology that $d$ generates, call it $q$, is generated by the closures of points and every open set is infinite. $(\mathfrak{A}, q)$ also is not a $T_{0}$-space since the closure of a point, which is infinite, is the closure of any point in it.

Now we will construct the counterexample using the following theorem.

THEOREM 3. There is a topology $q^{\#}$ such that $\left(\mathfrak{A}, q^{\#}\right)$ is a $T_{0}$-topology and $(\mathfrak{A}, q) \equiv_{\Delta(\mathfrak{E}(Q))}\left(\mathfrak{A}, q^{\#}\right)$.

Proof. To show this result we expand $\mathfrak{A}$ and $L$ to $\mathfrak{A}^{\#}$ and $L^{\#}$ as in Theorem 1 (taking pains to add functions to the language to pick out noninterior points from definable nonopen sets as in [3]).

Given a pair $a, b$ we will define a topology $q_{\langle a, b\rangle}$ such that $\left(\mathfrak{H}^{\#}, q\right)$ $\prec_{\mathscr{L}(Q)}\left(\mathfrak{I}^{\#}, q_{\langle a, b\rangle}\right), a$ and $b$ have unequal closures, and $q_{\langle a, b\rangle}$ is generated by the closures of points and every open set is infinite. This is the same topological property of $(\mathfrak{A}, q)$ which we use.

We then iterate this construction through all distinct pairs and take the union (see [3]) which will be $T_{0}$ and satisfy the conclusion to the theorem.

Define $x_{-1}=a$ and $y_{-1}=b$. Take $h$ to be a bijection from $\mathbf{N}$ into $\mathbf{N} \times \mathbf{N} \times 2$. Let $\psi_{i}(x), i \in \omega$, enumerate the $L_{A}^{\#}(Q)$ definable nonopen sets and let $\theta_{i}, i \in \omega$, enumerate the closures of points, which is a basis for $q$.

Assume we have defined $x_{-1}, \ldots, x_{n-1}, y_{-1}, \ldots, y_{n-1}$. We now will define $x_{n}$ and $y_{n}$. 
Assume $(h(n))_{2}=0$. Pick an $x$ such that $x \neq y_{i},-1<i<n-1$, and,

$$
x \in\left[\psi_{(h(n))_{1}}(x)\right]^{\left(\Re^{*}, q\right)} \text { and } \theta_{(h(n))_{0}} \subseteq\left[\psi_{(h(n))_{1}}(x)\right]^{\left(\Re^{*}, q\right)}
$$

or

$$
x \in \Theta_{(h(n))_{0}}-\left(\left[\psi_{(h(n))_{1}}(x)\right]^{\left(x^{*}, q\right)} \cup\left\{y_{i-1}\right\}_{0<i<n}\right),
$$

and set $y_{n}=y_{n-1}$ and $x_{n}=x$.

Otherwise pick a

$$
y \in \mathcal{O}_{(h(n))_{0}} \cap\left(\left[\psi_{(h(n))_{1}}(x)\right]\right)^{\left(\Re^{*}, q\right)}-\operatorname{Int}\left(\left[\psi_{(h(n))_{1}}(x)\right]^{\left(\Re^{*}, q\right)}\right)
$$

and

$$
x \in\left[\psi_{(h(n))_{1}}(x)\right]^{\left(\mathfrak{X}^{*}, q\right)}
$$

and set $y_{n}=y, x_{n}=x$, where $\operatorname{Int}(X)$ is the interior of the set.

If $(h(n))_{2}=1$ then switch $x$ and $y$.

This definition is possible because if

$$
\Theta_{(h(n))_{0}} \nsubseteq\left[\psi_{(h(n))_{1}}(x)\right]^{(\mathfrak{M}, q)},
$$

and

$$
\Theta_{(h(n))_{0}}-\left(\left[\psi_{(h(n))_{1}}(x)\right]^{\left(\mathfrak{X}^{*}, q\right)} \cup\left\{y_{i-1}\right\}_{0<i<n}\right)=\varnothing,
$$

then $\theta_{(h(n))_{0}}-\left[\psi_{(h(n))_{1}}(x)\right]^{\left(\mathscr{A}^{*}, q\right)}$ is nonempty and finite. Take a $y^{\prime} \in \theta_{(h(n))_{0}}-$ $\left[\psi_{(h(n))_{1}}(x)\right]^{\left(\mathscr{I}^{*}, q\right)}$. Then $\mathrm{Cl}\left(y^{\prime}\right) \cap \mathcal{\theta}_{(h(n))_{0}}$ is open, infinite and contains $y^{\prime}$. Hence there is a $y$ such that

$$
y \in \mathcal{O}_{(h(n))_{0}} \cap\left(\left[\psi_{(h(n))_{1}}(x)\right]^{\left(\Re^{*}, q\right)}-\operatorname{Int}\left[\psi_{(h(n))_{1}}(x)\right]^{\left(\mathfrak{x}^{*}, q\right)}\right) .
$$

Let $\theta=\left\{x_{i-1}\right\}_{i \in \omega}$ and $q_{\langle a, b\rangle}$ be the topology generated by $q, \theta$, and $\mathbf{N}-\theta . \theta$ and $\mathbf{N}-\theta$ are infinite because both of the sets $\left\{m \mid(h(n))_{2}=0\right\}$ and $\left\{n \mid(h(n))_{2}=\right.$ 1) are infinite.

Now $a \in \theta$ and $b \notin \theta$ and each set is infinite so all we need to show is that $\left(\mathfrak{A}^{\#}, q\right) \prec_{\mathfrak{R}(Q)}\left(\mathfrak{I}^{\#}, q_{\langle a, b\rangle}\right)$.

We show this by induction on the number of occurrences of $Q x$. Since $q \subseteq q_{\langle a, b\rangle}$ we need only to show one direction. So assume that $\left(\mathfrak{A}^{\#}, q_{\langle a, b\rangle}\right) \vDash Q x \varphi(x)$ and $\left(\mathfrak{I}^{\#}, q\right) \vDash \neg Q x \varphi(x)$ and derive a contradiction. Hence

$$
[\varphi(x)]^{\left(\mathfrak{\Re}^{*}, q_{\langle a, b\rangle}\right)}=[\varphi(x)]^{\left(\mathfrak{A}^{*}, q\right)}=\left(\mathcal{\Theta}_{\alpha} \cap \mathcal{\theta}\right) \cup\left(\mathcal{\Theta}_{\beta} \cap \mathcal{\theta}^{c}\right) \text {. }
$$

Either $\theta_{\alpha}$ or $\theta_{\beta}$ is not a subset of $[\varphi(x)]^{\left(\mathscr{a}^{*}, q\right)}$ since otherwise $\theta_{\alpha} \cup \theta_{\beta}=$ $[\varphi(x)]^{\left(\mathfrak{\Theta}^{*}, q\right)}$. So assume $\theta_{\alpha} \nsubseteq[\varphi(x)]^{\left(\Theta^{*}, q\right)}$ since the other case follows by symmetry.

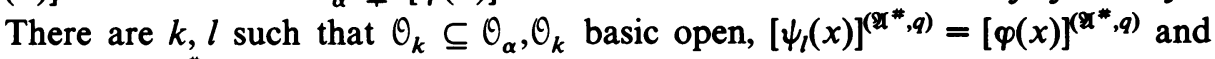
$\vartheta_{k} \nsubseteq\left[\psi_{l}(x)\right]^{\left(\Re^{*}, q\right)}$. Take $h^{-1}(\langle k, l, o\rangle)=m$ and we have

$$
\vartheta_{k}-\left(\left[\psi_{l}(x)\right]^{\left(\mathscr{O}^{*}, q\right)} \cup\left\{y_{i-1}\right\}_{0<i<m}\right)=\varnothing
$$

since otherwise $\theta_{\alpha} \cap \theta \nsubseteq[\varphi(x)]^{\left(\mathscr{A}^{*}, q\right)}$. Thus

$$
y_{m} \in\left(\theta_{k} \cap\left[\psi_{l}(x)\right]^{\left(x^{*}, q\right)}-\operatorname{Int}\left[\psi_{l}(x)\right]^{\left(\varkappa^{*}, q\right)}\right) \text {. }
$$

If $\theta_{\beta}=\varnothing$ then we are done since $y_{m} \notin \theta_{\alpha} \cap \theta$. 
To finish assume $\theta_{\beta} \neq \varnothing$. Then $y_{m} \in \theta_{\beta} \cap \theta^{c}$. Hence $y_{m} \in \theta_{\beta} \cap \theta_{k} \subseteq$ $\left[\psi_{l}(x)\right]^{\left(\mathfrak{R}^{*}, q\right)}$, since if

$$
\vartheta_{\beta} \cap \theta_{k} \cap\left\{y_{i-1}\right\}_{0<i<m}-\left[\psi_{l}(x)\right]^{\left(\mathfrak{x}^{*}, q\right)} \neq \varnothing
$$

then $\vartheta_{\beta} \cap \vartheta_{k} \cap \theta^{c}-\left[\psi_{l}(x)\right]^{\left(\mathscr{\Re}^{*}, q\right)} \neq \varnothing$ which is a contradiction. But $y_{m}$ is a noninterior point by definition so we have a contradiction.

We have shown the result and we can prove analogously the same result for $\Delta\left(\mathbb{L}\left(Q^{n}\right)_{n \in \omega}\right)$ via the same method.

\section{REFERENCES}

1. K. J. Barwise, Axioms for abstract model theory, Ann. Math. Logic 7 (1974), 221-265.

2. J. A. Makowsky and S. Shelah, The theorems of Beth and Craig in abstract logic, Trans. Amer. Math. Soc. 256 (1979), 215-239.

3. J. A. Sgro, Completeness theorems for topological models, Ann. Math. Logic. 11 (1977), 173-193.

4. , Completeness theorems for continuous functions and product topologies, Israel J. Math. 25 (1976), 249-272.

5. The interior operator logic and product topologies, Trans. Amer. Math. Soc. (to appear).

6. S. Willard, General topology, Addison-Wesley, Reading, Mass., 1970.

School of Mathematics, Institute for AdVanced Study, Princeton, New Jersey 08540

Current address: 12323 Brookshire Avenue, Downey, California 90242 